\title{
Possibilities and drawbacks of total reflection X-ray fluorescence spectrometry as a fast, simple and cost-effective technique for multielement analyses of cosmetics
}

\author{
E.Marguía, ${ }^{\mathrm{a},}$, R. Dalipi ${ }^{\mathrm{b}}$, L.Borgese ${ }^{\mathrm{c}}$, L.E. Depero ${ }^{\mathrm{c}}$, I.Queralt ${ }^{\mathrm{d}}$ \\ ${ }^{a}$ Department of Chemistry, University of Girona, C/M. Aurèlia Campmany, 69, 17003 Girona, Spain \\ b Instituto Zooprofilattico Sperimentale della Lombardia e dell'Emilia Romagna, B. Ubertini, 25124, \\ Brescia, Italy. \\ ${ }^{c}$ Department of Mechanical and Industrial Engineering, University of Brescia, 25123 Brescia, Italy. \\ ${ }^{\mathrm{d}}$ Institute of Environmental Assessment and Water Research, IDAEA-CSIC, Jordi Girona 18-26, 08034 \\ Barcelona, Spain
}

\begin{abstract}
This paper shows a simple, rapid and cost-effective method for multielement analyses of cosmetics. Total reflection X-ray fluorescence spectrometry (TXRF) is used to determine the composition, particularly the presence of potentially toxic elements, of cosmetics. Three sample preparation methods based on suspension preparation were developed for different types of cosmetic samples, e.g. lipsticks, eye shadows and body creams.

Limits of detection within the low $\mathrm{mg} \mathrm{kg}^{-1}$ range were obtained and enabled cosmetic screening for compliance with the legal thresholds for some major toxic elements. A good accuracy of the results on a wide range of concentration levels $\left(>10000 \mathrm{mg} \mathrm{kg}^{-1}\right)$ was found without the need of sample dilution. Results obtained for most elements in the lipstick and cream samples agreed with those obtained by the reference method recommended by the Food and Drug Administration, based on using inductively coupled plasma techniques after microwave digestion. However, for eyeshadow analysis, a more sophisticate approach is needed to improve the analytical results.
\end{abstract}

Keywords: TXRF; eye shadow; lipstick; cream; multielement; suspension

\footnotetext{
*Corresponding author: eva.margui@udg.edu, Tel: (+34) 648452182, ORCID: 0000-0003-4630-1530
} 


\section{Introduction}

Cosmetics have always been used by people worldwide since ancient times to clean, improve or change the appearance of skin, hair, nails and teeth. Unfortunately, cosmetics use is sometimes related to unfavourable effects resulting from the presence of chemical substances in these preparations, such as metals. Metals present in cosmetics may come from different sources, including the raw materials used in their production, the use of metal-coated equipment during manufacturing, and the intentional use as pigments or UV filters [1]. For filters, it is interesting to highlight the recent use of titanium dioxide $\left(\mathrm{TiO}_{2}\right)$ and zinc oxide $(\mathrm{ZnO})$ nanoparticles in sunscreens to absorb UVA/UVB radiation as an alternative to chemical UV filters [2].

The data available in the literature show that metals are present in different kinds of cosmetics (i.e., lipsticks, eye shadows and creams, etc.) at varying concentrations from almost undetectable values $\left(<\mu \mathrm{g} \mathrm{g}^{-1}\right)$ to a few grams of metal per kilogram of sample $\left(\sim \mathrm{g} \mathrm{kg}^{-1}\right)$ [3-6]. As metals are harmful for human health [7], their use in cosmetic product manufacturing is restricted or prohibited in many countries. The Food and Drug Administration (FDA) and the European Parliament (Regulation No. 1223/2009) have established international limits for metal impurities in cosmetics to prevent overexposure to metal ions. It is worth highlighting that even when an element is prohibited to produce cosmetics, this does not necessarily mean that the element is not present in the end product in detectable amounts. Therefore, in order to ensure consumer health, is it vital to employ adequate analytical techniques to monitor metals in cosmetic products.

The quantification of multiple elements in cosmetics is not easy, partly because of the wide range of element concentrations (from $\mu \mathrm{g} \mathrm{kg}^{-1}$ to $\mathrm{g} \mathrm{g}^{-1}$ ) and the complex matrix. For instance, lipsticks are composed of many ingredients, including waxes, oils, dyes and pigments. Pigments may include refractory minerals, such as alumina, silica, titanium dioxide and mica [8], which hamper chemical analyses. Several analytical methods have been developed and proposed for cosmetic analyses [9]. Table 1 summarises the analytical details of representative publications on the analyses of lipsticks, eye shadows and body creams. A common practice is the use of an extraction process of metals from the cosmetic matrix by microwave (MW) digestion, followed 
by atomic absorption spectroscopy (AAS) or inductively coupled plasma (ICP)-based techniques. The metal extraction rate strongly depends on the experimental conditions, such as sample weight, combination of acids, temperature and decomposition procedure [17]. As shown in Table 1, a mixture of an inorganic acid (i.e., $\mathrm{HNO}_{3}, \mathrm{HCl}, \mathrm{H}_{2} \mathrm{SO}_{4}, \mathrm{HClO}_{4}$ ) and an oxidant agent, such as $\mathrm{H}_{2} \mathrm{O}_{2}$, is normally used to carry out MW digestion. However, for those cosmetics containing refractory materials, the use of more aggressive reagents, such as HF with subsequent $\mathrm{H}_{3} \mathrm{BO}_{3}$ treatment, is required to achieve complete sample digestion $[8,11]$. In some applications, more complex sample treatments (i.e. solid-phase extraction on multi-walled carbon nanotubes) are necessary after the digestion process to enable the determination of elements present at ultratrace concentration levels by ICP optical emission spectrometry [18]. Very few papers have studied the direct analysis of cosmetic samples by slurry preparation due to matrix effect problems arising during the atomic spectroscopic analysis. For instance, to obtain quantitative results for several element determinations in cream samples by slurry preparation and an ICP-MS analysis, isotope dilution and standard addition methods are required [19]. Another cosmetic analysis option is the use of solid state techniques, such as laser-induced breakdown spectroscopy (LIBS) [16] or X-ray fluorescence spectrometry (XRF) $[7,20]$. Table 1 show that one of the major benefits of such techniques is a considerable reduction of the reagents, efforts and time required to prepare cosmetic samples before the analysis. However, the main drawback of LIBS and XRF methods is the use of adequate standards, with a similar matrix to the samples, for quantification purposes. All this is critical for cosmetic analyses because suitable cosmetic reference materials are lacking [22]. To this end, synthetic standards prepared in the laboratory by spiking procedures have been used as potential standards in quantitative analyses.

A less explored technique for cosmetic analyses is total reflection X-ray fluorescence spectrometry (TXRF). TXRF allows solid samples to be directly analysed by suspensions, but with a simpler quantification approach. As the sample in TXRF is deposited on a reflective carrier as a thin layer, the matrix effects commonly present in conventional XRF analyses are negligible and quantification can be carried out by internal standardisation without having to 
resort to calibration standards [23]. Despite the fact that this technique has been successfully applied for multielement analysis of solid samples in many fields such as soils [24-25], foodstuffs [26], biological samples [27], cultural heritage samples [28] and pharmaceutical samples [29], its use in the cosmetic analysis field has barely been explored.

In view of this premise, and taking into account that the quality control of cosmetic products requires many analyses per year, the main aim of this work was to study the possibilities and drawbacks of different methods based on suspension preparation and TXRF for the multielement analyses of different cosmetic types, including lipsticks, eye shadows and creams. In order to obtain the best conditions to carry out a cosmetic analysis by TXRF, different experimental parameters related to sample preparation and measurement conditions were evaluated. The developed methodologies were applied to analyse several commercial cosmetic samples, and the results were compared with those obtained by the reference method recommended by the FDA based on using ICP techniques after MW digestion.

\section{Materials and Methods}

\subsection{Reagents and materials}

Stock solutions of $1,000 \mathrm{mg} \mathrm{L}^{-1}$ (ROMIL PrimAg® Mono-component reference solutions) were used to prepare $\mathrm{Pd}, \mathrm{Rh}$ and Mo internal standard (IS) solutions and metal standards for ICPOES/ICP-MS calibration. Ultrapure deionised water (Millipore Corp., Bedford, Massachusetts, USA) was used to dilute the aforementioned stock solutions and cosmetic sample digests. A stock solution of $1,000 \mathrm{mg} \mathrm{kg}^{-1}$ of Mo in an oil matrix (High-Purity standards, USA) was used as the IS in the TXRF analysis of cosmetic samples with a high percentage of fat (i.e. lipsticks). This metal-organic standard provides high solubility and can be easily employed as an IS for organic matrices. All the organic solvents used throughout this work (Methylbenzene, diethylether, chloroform and carbon tetrachloride) were of analytical grade quality (SigmaAldrich, Spain). Triton ${ }^{\mathrm{TM}}$ X-100 (laboratory grade, Sigma-Aldrich) was used to prepare the cosmetic sample suspensions. Nitric acid (69\%, HIPERPUR, Panreac, Spain), hydrogen 
peroxide solution ( $\geq 30 \%$, TraceSELECT ${ }^{\circledR}$, Sigma-Aldrich, Spain), hydrofluoric acid $(47 \%$, TraceSELECT®, Sigma-Aldrich, Spain) and boric acid (Trace metal basis, Sigma-Aldrich, Spain) were employed for the microwave digestion procedures. Quartz glass disks $(30 \mathrm{~mm}$ diameter and $3 \mathrm{~mm} \pm 0.1 \mathrm{~mm}$ thick; Bruker-AXS, Germany) were used as sample holders to perform the TXRF analysis. To avoid damaging the quartz reflector surface when depositing digested samples that contain traces of hydrofluoric acid, reflectors with the same dimensions but made of acrylic glass (Bruker-AXS, Germany) were employed instead. A silicone solution in isopropanol (Serva $\mathrm{GmbH} \& \mathrm{Co}$, Germany) was used to coat all the quartz glass disc reflectors to obtain a hydrophobic film to facilitate sample deposition.

\subsection{Description of the cosmetic samples}

As suitable cosmetic certified reference materials were lacking, a set of lipstick and eye shadow samples available in Spanish markets was used to evaluate the analytical performance of the developed TXRF methods. The reference concentration values for the elements of interest were determined by microwave digestion and an ICP-OES/ICP-MS analysis (see Sections 2.3 and 2.5 for details). In order to explore the possibilities of the developed methods for a wide range of cosmetic sample analyses, the lipstick and eye shadow samples with different colours and pigments were considered: lipsticks (purple, red, brown, orange) and eye shadows (pink, orange, brown and blue). In the TXRF analysis of creams, the reference material CHEK RM 619 (body cream) was employed. This reference material certifies the content of several metals and metalloids ( $\mathrm{Pb}, \mathrm{Cd}, \mathrm{Cr}, \mathrm{Ni}, \mathrm{Hg}, \mathrm{Sb}$ and $\mathrm{Se})$ at trace levels [30]. Several body cream samples available in Spanish markets were also considered to study the real capabilities of the developed method for body cream analyses.

\subsection{Sample suspension for the TXRF analysis}

Preliminary studies were performed to select the best conditions to prepare the lipstick, eye shadow and cream suspensions for the TXRF analysis (see Sections 3.1, 3.2 and 3.3 for further details). Table 2 summarises the suspension preparation for each cosmetic type. As we can see, 
the general procedure for all cosmetic types involved suspending several $\mathrm{mg}$ of sample in an adequate disperser agent. Homogeneous suspensions were obtained for the eye shadow and cream suspensions using a solution of Triton $1 \%$ in water, but an organic solvent (chloroform) was employed for lipsticks because of their high fatty material content (including waxes and oils). In all cases, quantification was performed by means of internal standardization, thus a suitable IS was added to the suspension before the TXRF analysis. In view of the large number of elements present in cosmetic samples within the $2-18 \mathrm{keV}$ energy range, and taking into account the possibility of detecting high $\mathrm{Z}$ elements through its $\mathrm{K}$-lines by the TXRF system (equipped with a ${ }_{74} \mathrm{~W}$ X-ray tube), $\mathrm{Pd}$ and Mo were selected as the IS. The usefulness of high-Z elements as the IS has been demonstrated in previous studies using an ${ }_{74} \mathrm{~W}$ X-ray tube based TXRF instrumentation [28]. As reported in Table 2, for the specific case of lipstick samples, a Mo solution of $200 \mathrm{mg} \mathrm{L}^{-1}$ in an oil matrix was employed for internal standardization instead of the more widely used aqueous standards due to the organic nature of the lipstick matrix. To increase the homogeneity of the resulting cosmetic suspensions, a sonication process was carried out before depositing $5 \mu \mathrm{L}$ of sample on a quartz reflector. An IR lamp was used to cut the time required to dry the spot deposited on the reflector. No heating process was necessary with the lipstick samples because of the high volatility of the organic solvent used to suspend samples, and the drying process was run at room temperature in a laminar flow hood.

It is noteworthy that for all the studied cosmetic samples, the complete sample treatment procedure was simple, quite fast and took less than 10 minutes to obtain suitable specimens to be analysed by TXRF.

\subsection{Sample digestion for TXRF and ICP analyses}

As pointed in the Introduction section, a wide range of digestion methods are used for cosmetic sample treatments (see Table 1). The present work employed a slightly modified version of the digestion method proposed by Hepp and co-workers [8], recommended by the FDA. Briefly: an $0.2 \mathrm{~g}$ aliquot of cosmetic sample (lipstick, eye shadow or cream) was weighed in a Teflon vessel and was allowed to come into contact with $7 \mathrm{~mL}$ of $\mathrm{HNO}_{3}, 2 \mathrm{~mL} \mathrm{HF}$ and $2 \mathrm{~mL}$ of $\mathrm{H}_{2} \mathrm{O}_{2}$. Then 
vessels were capped and heated following a four-stage microwave digestion programme using an Ethos Plus Milestone microwave with an HPR-1000/10S high pressure rotor (Sorisole, Bergamo, Italy). Step-1: 15 minutes to reach $130^{\circ} \mathrm{C}$, Step-2: 3 minutes at $130^{\circ} \mathrm{C}$, Step-3: 15 minutes to reach $200^{\circ} \mathrm{C}$ and Step-4: 30 minutes at $200^{\circ} \mathrm{C}$. Extracts were also treated with $1 \mathrm{~g}$ of boric acid, and they were heated for $10 \mathrm{~min}$ at $180^{\circ} \mathrm{C}$ to allow fluoride complexation and to avoid damaging the quartz torch in the ICP analysis. Finally, digests were transferred to $25 \mathrm{~mL}$ flasks and were brought to volume with ultrapure deionised water.

The resulting sample digests were directly analysed by the ICP-OES and ICP-MS techniques (see Section 2.5 for the measurement details). In the case of TXRF analysis, a $1 \mathrm{~mL}$ aliquot from each sample digest was fortified with a suitable volume of an aqueous $200 \mathrm{mg} \mathrm{L}^{-1} \mathrm{Pd}$ solution (IS) to obtain a final concentration of $10 \mathrm{mg} \mathrm{L}^{-1}$. Then $5 \mu \mathrm{L}$ of the resulting solution were deposited on an acrylic glass reflector, dried under an IR lamp and analysed by TXRF.

\subsection{Multielement analysis by the TXRF and ICP-based techniques}

TXRF analysis of cosmetics, both digested and suspended samples, was performed in a commercial benchtop spectrometer "S2 PICOFOX ${ }^{\mathrm{TM} " ~(B r u k e r ~ N a n o ~ A n a l y t i c s, ~ B e r l i n, ~}$ Germany). This system is equipped with a low-power ${ }_{74} \mathrm{~W}$ X-ray tube that allows TXRF analyses to be performed using K-lines of high atomic-number elements. Compared to other existing systems, another advantage of this spectrometer is that no cooling media and gas consumption are required for it to function. Table S1 presents the main instrumental characteristics and measuring conditions used. The evaluation of the TXRF spectra and the calculation of the analyte net peak areas were performed with the equipment's software (Spectra Plus 5.3, Bruker AXS Microanalysis GmbH, Berlin, Germany). With this software, the spectral background, composed of the detector shelf and scattered excitation radiation, is calculated by a mathematical algorithm and subtracted from the spectrum. To allocate the correct intensities to the individual elements if individual florescence peaks considerably overlap, the software applies a deconvolution routine (SuperBayes), which uses the measured mono-element profiles to evaluate peak intensities. 
Quantitative analyses were carried out by internal standardization (no external calibration was needed) by using Expression (1) [23]:

$C_{i}=\left(\frac{N_{i} C_{p i} S_{p i}}{N_{p i} S_{i}}\right)$

Expression (1)

where $\mathrm{C}_{\mathrm{i}}$ is the analyte concentration, $\mathrm{N}_{\mathrm{i}}$ if the analyte net peak area, $\mathrm{C}_{\mathrm{pi}}$ is the IS concentration, $\mathrm{S}_{\mathrm{pi}}$ is the instrumental sensitivity for the IS, $\mathrm{N}_{\mathrm{pi}}$ is the IS net peak area and Si is the instrumental sensitivity for the analyte.

To evaluate the capabilities of the developed TXRF method for the trace analysis, limits of detection (LoD) were calculated by Expression (2):

$L o D=\frac{3 \mathrm{C}_{\mathrm{i}} \sqrt{\mathrm{N}_{\mathrm{bkg}}}}{\mathrm{N}_{\mathrm{i}}}$

Expression (2)

where $C_{i}$ is the concentration of a given analyte; $N_{b k g}$ is the background count rate and $N_{i}$ is the net count rate of the analyte. This equation is analogous to the $3 \sigma$ definition of LoDs [23].

For comparison purposes, the digested cosmetic samples were also analysed by ICP-OES (for element concentrations at the $\mathrm{m} \mathrm{L}^{-1}$ level) and ICP-MS (for element concentrations at the $\mu \mathrm{g} \mathrm{\textrm {L } ^ { - }}$ ${ }^{1}$ level). The instrumental parameters and measurement conditions for both ICP systems are displayed in Table S1. It was noteworthy that to ensure the good quality of the results obtained by the ICP measurements, control multielemental standards were analysed daily and a careful evaluation of spectral interferences was carried out. 


\section{Results and Discussion}

\subsection{TXRF analysis of lipsticks}

\subsubsection{Method development}

In view of the micro-analytical and multi-analytical capabilities of TXRF [23], qualitative information about the elements present in the lipstick samples can be easily obtained by covering the reflector with a thin layer of the target sample (with no sample treatment). Figure 1 depicts the TXRF spectra in the lipstick samples analysis obtained by this analytical approach. As shown, different TXRF spectrum shapes were obtained depending on the lipstick type. For instance, the orange lipstick is characterised by the presence of iron and bromine, whereas the presence of barium is characteristic in the purple and red lipsticks. This information can be very useful since the elements present in lipsticks can be related to the pigments used to add colour to the lipstick base matrix.

However, if the objective is to quantify the elements present in lipstick samples, then further sample treatments are needed. Digestion is still the most widespread procedure to treat solid samples before a TXRF analysis. A recent review focused on sample pre-treatment strategies for TXRF analyses highlighted that the suspension preparation of solid samples (without digestion) accounts for only $15 \%$ of the followed sample treatment procedures [31]. Nevertheless, recent publications have demonstrated the suitability of this analytical approach for the quantitative determination of silver nanoparticles in soil samples [25], and the multielement analysis of plant foodstuff [26] and biological samples [27]

In an attempt to develop a simple, fast and cost-effective TXRF method to analyse cosmetics, the possibility of using cosmetic suspensions as a sample preparation strategy for TXRF analyses was explored. Firstly, a careful evaluation of the analytical conditions used for suspension preparations was made, including the disperser agent type, the amount of sample used to prepare suspensions, the sample deposition volume and the drying mode of the drop on the reflector. 
Considering the organic nature of lipsticks, several organic solvents were tested to prepare the suspension, such as methylbenzene, diethyl ether, chloroform and carbon tetrachloride. Our previous works demonstrated the usefulness of chlorine-based solvents in microextraction procedures combined with a TXRF analysis. The main advantage of these organic solvents is their high volatility, which facilitates the drying step of the resulting solution on the reflector [32-33]. Other dispersing agents normally used to prepare solid samples (deionised water, a dilution of $1 \%$ Triton X-100 and a dilution of 5\% Triton X-100) [34-36] were discarded herein because it was impossible to obtain a homogeneous suspension of the lipstick sample. Finally, chloroform was selected as the best solvent in suspension homogeneity terms despite its toxicity because only a small volume of this solvent was required to prepare the suspension.

The amount of sample used for suspension preparation purposes can influence the TXRF analysis as it is related to the thickness of the deposited sample on the reflector and, therefore, to the total reflection condition of the incident X-ray beam, which is a prerequisite for a proper TXRF analysis [23]. Figure S1 (A) reflects the effect of the amount of sample used to prepare lipstick suspensions on certain element concentrations. As a study case, experimental tests were performed using the red lipstick sample. For comparison purposes, the elemental concentrations determined by ICP-OES and ICP-MS are also displayed in Figure S1 (A) as reference values. As shown, a greater deviation of replicate measurements was achieved by using $5 \mathrm{mg}$ of sample. This was probably due to the lack of homogeneity and representativeness from using such a small amount of the original sample. As no significant differences were observed in terms of element concentrations using $10 \mathrm{mg}$ or $20 \mathrm{mg}$ of sample, $10 \mathrm{mg}$ was selected as the sample amount to prepare the lipstick suspensions.

One critical issue when preparing solid suspensions in TXRF analysis is the sedimentation of the solid sample at the bottom of an Eppendorf tube, which leads to non-homogeneous sample suspension. For this reason, a mixing procedure is often required before depositing the sample on the reflector. The experimental tests demonstrated that the best way to obtain a homogenous suspension for the lipstick samples was a sonication process $(5 \mathrm{~min})$, followed by mixing the resulting samples by vortex agitation (2 min). 
As stated in the Introduction section, one of the advantages of TXRF is the possibility of running multielement quantitative analyses by internal standardization without needing external calibration. The last point is feasible, provided that the sample is deposited on a reflective carrier as a thin layer $(<100 \mu \mathrm{m})$. In this case, matrix effects are negligible and the element concentrations in the sample can be determined by adding a suitable IS to the sample and considering the instrumental sensitivities of analytes and the IS [23]. By considering this fact, the sample deposition volume on the reflector is a critical parameter to be tested for a proper TXRF analysis. Figure S1 (B) presents the elemental concentrations obtained by depositing 5 $\mu \mathrm{L}$ and $10 \mu \mathrm{L}$ of the suspended red lipstick sample under the aforementioned conditions. In general, no significant differences in the results were obtained between the tested sample volumes. However when $5 \mu \mathrm{L}$ were used, the resulting drop was deposited better on the sample carrier. Consequently, this volume was fixed for further tests.

After sample deposition, the resulting drop needs to be dried before the TXRF analysis. This tends to be done by a heating process using an IR lamp or a hot-plate. However, given the high volatility of the solvent used to prepare the lipstick suspensions, it was not necessary to apply any heating source and the resulting drop was dried at room temperature in a laminar flow hood in less than 1 minute.

The measurement time can also affect the repeatability of the results, above all for the determination of the trace elements. Figure 2 offers an example of the relative standard deviation (RSD) for a trace element $(\mathrm{Rb})$ and a minor element (Ti) obtained by analysing a suspension of the red lipstick sample 5 times. As we can see, the effect of the measurement time on the repeatability of the results was not significant (RSD: 6-3\%) for those elements present at higher concentrations (i.e., $\mathrm{Ti} \sim 6000 \mathrm{mg} \mathrm{kg}^{-1}$ ). However, for the elements present at trace levels (i.e., $\mathrm{Rb} \sim 60 \mathrm{mg} \mathrm{kg-1)}$, a longer measurement time (2000s) was necessary to obtain a similar repeatability of the results. Since the aim of this application was to carry out a multielement analysis of the lipstick samples, including minor and trace elements, a measurement time of $2000 \mathrm{~s}$ was selected as a trade-off between acceptable repeatability and the total analysis time. It is interesting to remark that the measurement time can be cut if the objective is to determine the 
elements present at thousands of $\mathrm{mg} \mathrm{kg}^{-1}$ or if TXRF systems equipped with ${ }_{42} \mathrm{Mo} \mathrm{X}$-ray tubes are used. It has already been demonstrated that a measuring time can be cut from $2000 \mathrm{~s}$ to $600 \mathrm{~s}$ to achieve similar analytical performance with such systems [37].

\subsubsection{Analytical performance}

As suitable certified reference lipstick materials are lacking, a set of different coloured lipsticks was used to evaluate the analytical capabilities of the developed TXRF method. The reference concentration values for the elements of interest in these samples were determined by microwave digestion and an ICP-OES/ICP-MS analysis (see Section 2.4 for details). Figure 3 provides the concentration values obtained by TXRF and ICP. For comparison purposes, the results of analysing the lipstick sample digests by TXRF are also included.

In general, a good agreement was found between the results obtained by the TXRF analysis using suspension/digestion sample treatments, which highlights the possibilities of employing suspension preparation as a simpler, faster and cost-effective analytical approach to analyse lipsticks by TXRF. Even some elements (i.e., Ca, Mn, Zn, Sn and Ba) can be quantified only by TXRF after suspension preparation. As stated in Section 2.4, the sample dilution factor of the digestion procedure is quite high $(\sim 150)$, which leads to high LoDs in the solid sample. In fact the LoDs for the mid-Z elements around $\sim 2 \mathrm{mg} \mathrm{kg}^{-1}$ were estimated for suspension preparation and the LoDs for the lipstick digests were 5-fold higher. According to our results, it was concluded that the combined suspension preparation and TXRF analysis approach was inadequate to monitor elements present at ultra-trace concentrations $\left(<1 \mathrm{mg} \mathrm{kg}^{-1}\right)$, but the obtained LoDs enabled us to screen if the lipstick samples complied with at least the legislation thresholds for some main toxic elements. For instance for $\mathrm{Pb}$, the limit set by the German Government is $20 \mathrm{mg} \mathrm{kg}^{-1}$ and the Canadian Government establishes a limit of $10 \mathrm{mg} \mathrm{kg}^{-1}$ [3839].

It is interesting to note that the LoDs for light elements (i.e. K, Ca) were around $100 \mathrm{mg} \mathrm{kg}^{-1}$ due to the low fluorescence field characteristic of these elements and the low energy of their 
analytical lines which is partly absorbed by air. Nevertheless, this was not a critical point for the lipstick analysis because light elements were present at high concentration levels (see Figure 3). Regarding the comparison of suspension-TXRF and ICP analysis, similar results for determination of most mid-Z elements were obtained using both analytical approaches with recovery values in the range of $80-120 \%$. However, as it is shown in Figure 3, precision of the results (in RSD terms RSD of triplicate analysis) by suspension-TXRF analysis was significantly higher than in ICP analysis, with values around $15-20 \%$. We ought to bear in mind that only a small amount of sample $(10 \mathrm{mg})$ was used during suspension preparation to perform the analysis. Thus the sample homogeneity of the original cosmetic sample is a critical point to ensure a good precision and representativeness of the results.

In the case of $\mathrm{K}$ and $\mathrm{Ca}$, a systematic underestimation of the concentration values was obtained (recoveries $\sim 70 \%$ ). This can be explained by the absorption issues arising from the cosmetic matrix. A similar tendency has been reported recently when plant foodstuff suspensions were analysed by TXRF [26], and the results for these light elements can be improved by employing external calibration as the quantification approach instead of internal standardization. Hence other quantitative approaches should be explored for the quantitative determination of light elements in lipstick samples by suspension and TXRF analyses. As shown in Figure 3, inaccurate results were also obtained for $\mathrm{Mn}$ and $\mathrm{Ba}$ determinations in some analysed lipstick samples, with recovery values at around $>150 \%$, and $\sim 40 \%$, respectively. The determination of trace amount of $\mathrm{Mn}$ in samples containing large amounts $\left(>10,000 \mathrm{mg} \mathrm{kg}^{-1}\right)$ of Fe (i.e. in the red lipstick sample) was hampered by lines $\mathrm{Mn}-\mathrm{K}_{\beta}$ and $\mathrm{Fe}-\mathrm{K}_{\alpha}$ overlapping. The inaccuracies in the Ba concentrations can be explained by taking into account the substantial overlapping of the Ba$\mathrm{L}$ lines (used as the analytical lines) and $\mathrm{K}$-lines of $\mathrm{Ti}$, which is present in most lipstick samples at very high concentrations $\left(>1,000 \mathrm{mg} \mathrm{kg}^{-1}\right)$. To improve the analytical Ba determination results, another analytical line (i.e., $\mathrm{Ba}-\mathrm{K}_{\alpha}$ ) should be tested. To do so, a set of suitable $\mathrm{Ba}$ standards should be used to calibrate the ${ }_{74} \mathrm{~W}$ X-ray tube system. However, most TXRF systems are equipped with ${ }_{42} \mathrm{Mo} \mathrm{X}$-ray tubes and the determination of Ba K-lines is not possible. So the 
accurate $\mathrm{Ba}$ determination in lipstick samples with large amounts of $\mathrm{Ti}$ is a tricky issue in a TXRF analysis, which should be studied individually in future research.

The ICP-MS analysis of the studied lipstick samples confirmed the presence of $\mathrm{Co}, \mathrm{Ni}$ and $\mathrm{Cu}$ at concentration levels below the limits of quantification for the developed TXRF method. No other toxic and harmful elements, like $\mathrm{As}, \mathrm{Hg}, \mathrm{Pb}$ and $\mathrm{Cd}$, were detected in any considered lipstick.

\subsection{TXRF analysis of eye shadows}

\subsubsection{Method development}

Unlike the lipstick analysis, with the eye shadow suspensions, the sedimentation of the solid eye shadow particles is a critical issue, so it is more difficult to obtain a homogeneous suspension. Hence well selecting the amounts of the sample and disperser agents used to prepare slurry is of paramount importance. To do so, several experimental tests were run using different disperser agents and eye shadows amounts. Regarding the disperser, diluted solutions of commercial surfactant Triton X-100 were considered. The non-ionic nature of this surfactant improves the homogeneity of most solid suspensions, as clearly pointed out in other applications that have dealt with using Triton X-100 as a dispersing agent [34]. Employing double-deionised water was discarded because it was not possible to suspend eye shadow particles. The results obtained about the effect of the disperser agent and the amount of eye shadows on the determined element concentrations by TXRF are offered in Figure S2. Here we can see that similar results were obtained regardless of using the $1 \%$ Triton X-100 and 5\% Triton X-100 solutions. However, the background of the obtained TXRF spectra increased considerably by raising the surfactant concentration due to this product's organic matrix. Therefore, $1 \%$ Triton $\mathrm{X}-100$ was considered appropriate for further experiments. Regarding the amount of sample, better results were obtained when using larger sample amounts, except for low $\mathrm{Z}$ elements. Finally as a compromise, eye shadow suspensions were prepared by suspending $20 \mathrm{mg}$ of sample in $1 \mathrm{~mL}$ of $1 \%$ Triton X-100. 
As indicated before, one critical point of eye shadow suspensions is the sedimentation of solid particles before depositing the sample on the reflector. Therefore, several strategies were explored to cope with this fact, including a 5-minute sonication process in a conventional ultrasound bath and a 2-minute sonication step in a cup horn ultrasonicator device [40] set at $40 \%$ amplitude. As the optical images displayed in Figure S3 show, the shape and distribution of the residue on the reflector were not significantly different when the various considered approaches were followed. In all cases, most of the solid concentrated at the centre of the residue deposited on the reflector. Regarding spot size, it is noteworthy that when no sonication process was applied, the diameter of the deposited spot was $15 \%$ smaller than for the residues that resulted after a sonication step. Samples were analysed by $\mu$-XRF to study the distribution of the elements present in the deposited residue while preparing suspensions with and without a sonication process. Figure 4 displays the 2-D mappings for the $\mathrm{Cl}, \mathrm{K}, \mathrm{Ca}$ and Ti distributions in the eye shadow residues without sonication before deposition and with 5 minutes of conventional sonication. It was not possible to monitor the other trace elements present in the eye shadow samples due to the $\mu$-XRF system's limited sensitivity. As expected, elements accumulated in the central part of the reflector, where the eye shadow residue concentrated. Slightly better element distribution homogeneity in the residue was found when preparing the suspension after a 5-minute sonication step. So this sample preparation condition was set for further tests.

The experimental tests run to evaluate the effect of sample deposition volume on element concentrations determined in eye shadow samples were also carried out. Deposition of an overlarge sample volume on the reflector could lead to a thicker suspension residue. Thus the total reflection condition could break. Figure S4 (A) displays the results obtained for the analysis of $5 \mu \mathrm{L}$ and $10 \mu \mathrm{L}$ of an eye shadow suspension. Slightly better results were obtained with $5 \mu \mathrm{L}$ of sample and therefore, this volume was used in the following experimental tests. The heating mode used to dry the sample on the reflector surface did not significantly affect the obtained results (see Figure S4 (B)), so an IR lamp was employed to dry the eye shadow samples as the required drying time was shorter $(<2 \mathrm{~min})$. 
As stated in Section 3.1.1, measurement time is a parameter that can influence the repeatability of the obtained TXRF results, particularly for trace analyses. This was why an eye shadow sample was prepared as a suspension under the aforementioned analytical conditions aforementioned and it was analysed at different measurement times within the 500-2000 seconds range. It was found that for the elements present at high concentrations (i.e. $\mathrm{Ca} \sim 4,000$ mg $\mathrm{kg}^{-1}$ ), a measurement time of $1000 \mathrm{~s}$ was suitable to obtain an acceptable repeatability of the results (RSD 3\%). However, a longer measurement time was needed to obtain similar RSD values when determining trace elements $\left(<100 \mathrm{mg} \mathrm{kg}^{-1}\right)$. As the study aim was to develop an analytical method for a multielement analysis of eye shadows, including major, minor and trace elements, a measurement time of $2000 \mathrm{~s}$ was selected to carry out the TXRF analysis.

\subsubsection{Analytical performance}

A set of different coloured eye shadow samples was digested and analysed by ICP techniques. The determined elemental concentration values were used as references to evaluate the analytical capabilities of the TXRF method developed for the eye shadow analysis. Firstly, LoDs were estimated for both suspended and digested samples. As with the lipstick analysis, the LODs for the suspended samples were low $\mathrm{mg} \mathrm{kg}^{-1}$ (low $\mathrm{Z}$ elements: $\sim 150 \mathrm{mg} \mathrm{kg}^{-1}$, mid-Z elements: $\sim 5 \mathrm{mg} \mathrm{kg}^{-1}$, high $\mathrm{Z}$ elements: $\sim 10 \mathrm{mg} \mathrm{kg}^{-1}$ ) and were about 4-fold lower than those obtained by acid digestion due to the sample's high dilution factor and also to the lower reflectivity of the acrylic glass reflector used for the digested samples analysis. As we can see, LODs for the eye shadow sample analysis were slightly higher than those previously estimated for the lipstick analysis. This can be explained by the presence of small solid particles on the quartz reflector surface that resulted in a higher TXRF spectra background. By the proposed TXRF method, elements like K, Ca, Ti, Mn, Fe, Zn, Rb, Sr, Ba and Sn were identified in the studied eye shadow samples. The ICP-MS results also revealed the presence of small amounts of $\mathrm{Cr}$, $\mathrm{Co}$, Ni and $\mathrm{Cd}\left(3-7 \mathrm{mg} \mathrm{kg}^{-1}\right)$ in the brown eye shadow. No other toxic elements like As, $\mathrm{Se}, \mathrm{Sb}, \mathrm{Hg}$, were detected in any studied sample. 
Figure 5 summarises the results obtained by the eye shadow TXRF analysis and the ICP techniques. It shows a clear overestimation of the results obtained by the suspension-TXRF analysis (Recoveries $>120 \%$ ) and that a digestion of samples is needed before the TXRF analysis to obtain quantitative results. Therefore, despite the fact that the use of suspension preparation as a simple and cost effective analytical approach can be useful as screening tool to evaluate the composition of eyeshadows, including elements present at high concentration levels $1000-100000 \mathrm{mg} \mathrm{kg}^{-1}$, a normalization process is required to obtain quantitative data. One possibility is to use a calibration curve for each target element obtained by analysing a set of samples with a similar matrix to the unknown ones via a reference technique. Our previous publication demonstrated the suitability of this quantification strategy to improve TXRF results in a TXRF analysis with a human placenta sample [27]. Future research into eye shadow TXRF analyses will focus on this.

Another interesting aspect reflected by the results in Figure 5 is the presence of $\mathrm{Sn}$ in most analysed eye shadows. This element is not included in Regulation 1223/2009 of the European Parliament about elements prohibited, restricted or allowed in cosmetics. Despite the apparently low toxicity and harmful effects of inorganic species of Sn for humans, certain organotin compounds included in many applications used as biocides and antifungal agents can be as toxic as cyanide [41]. Therefore, monitoring $\mathrm{Sn}$ in some cosmetic products like eye shadows should at least be considered in the future. TXRF systems equipped with W X-ray sources should be used for such purpose. It is well-known that the determination of high $\mathrm{Z}$ elements using the most widespread ${ }_{42}$ Mo X-ray tube systems is hampered by the Sn L-lines and K-lines of light elements overlapping (e.g., $\mathrm{K}$ and $\mathrm{Ca}$ ) [37], which are present at high concentrations in most cosmetic samples.

The repeatability of the results of the TXRF method precision obtained by a triplicate analysis of eye shadow suspensions was similar to that associated with other atomic spectroscopic techniques, which mostly obtained RSD values below $5 \%$. 


\subsection{TXRF analysis of body creams}

\subsubsection{Method development}

With the cream samples, a commercially available reference material (Body Cream RM619) from the Netherlands Food Consumer Product Safety Authority was used to evaluate the best analytical conditions to carry out the TXRF analysis with these samples. This reference material certifies the content of several metals and metalloids $(\mathrm{Pb}, \mathrm{Cd}, \mathrm{Cr}, \mathrm{Ni}, \mathrm{Hg}, \mathrm{Sb}$ and $\mathrm{Se})$ at trace levels $\left(<100 \mathrm{mg} \mathrm{kg}^{-1}\right)$.

A set of experimental tests was firstly carried out to select the best way to prepare cream suspensions for the subsequent TXRF analysis. It was found that a good approach to prepare the cream suspension was to disperse $20 \mathrm{mg}$ of the sample in $1 \mathrm{~mL}$ of the $1 \%$ Triton $\mathrm{X}-100$ solution. As with the eye shadow samples, using double-deionised water was discarded as the disperser agent because it was impossible to obtain good cream suspension homogeneity. Higher surfactant solution concentrations (i.e., 5\% Triton X-100) were not considered in view of the high background of the obtained TXRF spectra, which hampers determining trace element concentrations. After preparing the suspension and before depositing the sample on the reflector, a 5-minute sonication step was run to improve cream suspension homogeneity.

The effect of the sample deposition volume with the cream analysis was also studied. To do so, the cream reference material was analysed in triplicate under the aforementioned conditions by using two different deposition volumes (5 and $10 \mu \mathrm{L})$. The obtained results are displayed in Figure S5, where better results were obtained in accuracy and precision terms when a $10 \mu \mathrm{L}$ aliquot of the cream suspension was used on the reflector. So this volume was selected for further tests.

As previously stated, the use of long measurement times $(>1000 \mathrm{~s})$ is crucial to obtain a good repeatability of the trace analysis results. For this reason, a measurement time of 2000 seconds was selected in view of the low concentration levels $\left(<100 \mathrm{mg} \mathrm{kg}^{-1}\right)$ of the analytes present in the reference cream material. 


\subsubsection{Analytical performance}

To check the analytical capabilities of the developed TXRF method to analyse the cream samples, the aforementioned reference material was prepared as a suspension under the analytical conditions described in Section 3.3.1 and analysed in triplicate. By way of example, Figure 6(A) displays the TXRF spectrum obtained by analysing one of the replicates. Apart from certified elements, other elements likes $\mathrm{Mn}, \mathrm{Fe}, \mathrm{Co}$ and $\mathrm{Zn}$ were identified in the body cream sample. The possibility of multielement qualitative information is one of the advantages of TXRF compared to other spectroscopic techniques used in the cosmetic analysis field.

Table 3 reports the LoDs and results obtained from analysing the body cream by TXRF. The reference values are also included for comparison purposes. We can see that a generally good agreement was obtained between the certified values and those determined by the developed TXRF method (Recoveries: 89-111\%). The widest divergences went to $\mathrm{Cr}$ and $\mathrm{Pb}$ (Recoveries $>115 \%$ ). Table 3 shows that these elements were present at concentration levels which came close to the method's limit of quantification, and that the accuracy and precision of the results were worse than for other considered elements. Surely, an improvement of the results for these elements could be assessed using a TXRF system equipped with a Mo X-ray tube. Previous studies have already demonstrated that the LoDs for the light-medium $\mathrm{Z}$ elements with $\mathrm{K}$-line detection (i.e. $\mathrm{Cr}$ ) and the high $\mathrm{Z}$ elements with $\mathrm{L}-$ line detection (i.e., $\mathrm{Pb}$ ) are 1 order of magnitude lower than the TXRF systems equipped with W X-ray tubes [37]. However, the use of a W X-ray tube herein was able to determine high $\mathrm{Z}$ elements (i.e, $\mathrm{Cd}, \mathrm{Sb}$ ) at low concentration levels (LODs: 1.6-2.8 $\mathrm{mg} \mathrm{kg}^{-1}$ ), which was not possible with ${ }_{42} \mathrm{Mo}$ TXRF systems due to the low sensitivity and overlapping issues of the employed Cd and Sb L lines. In Table 3, the $\mathrm{Hg}$ quantification in the reference body cream material was not possible by the developed TXRF method given the low concentration level $\left(2.33 \pm 0.81 \mathrm{mg} \mathrm{kg}^{-1}\right)$ and the problems of $\mathrm{Hg}$ volatilisation that occurred in the drying step on the reflector. To obtain reliable results for $\mathrm{Hg}$ determinations at trace and ultra-trace levels by TXRF, more complex sample treatments are needed to prevent $\mathrm{Hg}$ volatilisation [42-43], which was not the purpose of the present study. 
Finally from the results reported in Table 3, it is interesting to remark that the precision of the results achieved by the cream suspension TXRF analysis are acceptable (RSD: 2-12\%) and comparable to other spectroscopic methods that analyse such cosmetics.

To further explore the capabilities of the developed method for our intended purposes, a set of other body cream samples available in Spanish markets was also analysed.

Nevertheless only in one of the samples considered a significant peak of Ti was detected in the resulting TXRF spectrum (see Figure 6(B)). As reported in the literature, Ti is usually added to some cream products in the form of $\mathrm{TiO}_{2}$ as a UV filter or a whitening agent [2]. By the TXRF method, the Ti concentration estimated in this sample was $1,100 \pm 300 \mathrm{mg} / \mathrm{kg}$, which agrees with the reference concentration determined by digestion and the ICP-OES analysis $(1,126 \pm 10$ $\mathrm{mg} / \mathrm{kg}$ ). However as shown, a wide dispersion between replicates was obtained (RSD $25 \%$ ) by the TXRF method. This finding may be related to the inadequate Ti homogeneous distribution in the original sample, which dramatically affected the final TXRF results as only a small amount of sample (20 mg) was used to perform the cream analysis.

\section{Conclusions}

This work evaluated the possibilities and drawbacks of the combined use of suspension preparation and TXRF for a multielement analysis of different cosmetic types: lipsticks, eye shadows and body creams. An important part of the use of these methodologies is to understand and recognise their capabilities and limitations, and to evaluate their suitability and complementarity depending on the objective of the analyses and sample characteristics.

Applying the direct analysis of lipstick and body cream suspensions by TXRF could be a fast, simple and cost-effective methodology to acquire quantitative information about most of the mid-Z elements present in such samples. However for some specific elements like low-Z elements, $\mathrm{Ba}$ or $\mathrm{Hg}$, the use of other quantification approaches or sample treatments to obtain quantitative results have to be explored. LoDs in the low $\mathrm{mg} \mathrm{kg}^{-1}$ range can be obtained with this simple sample treatment combined with TXRF analysis. This fact limits the application of the methodology to monitor those elements present at ultratrace concentrations $\left(<1 \mathrm{mg} \mathrm{kg}^{-1}\right)$, but 
the provided LoDs enable cosmetic samples to be screened and at least comply with the legislation thresholds for some main toxic elements (i.e. the limit for $\mathrm{Pb}$ in lipsticks set by the German Government is $20 \mathrm{mg} \mathrm{kg}^{-1}$ ). With the proposed TXRF methods, the determination of those elements present at high concentration levels $\left(>10,000 \mathrm{mg} \mathrm{kg}^{-1}\right)$ is possible without having to resort to sample dilution, which is required in other spectroscopic techniques. Another interesting advantage of this analytical approach is the use of internal standardization for quantification purposes instead of external calibration when a set of matrix-matched standards is required for calibration purposes.

The quality of the results for the case eye shadow samples obtained by the suspension-TXRF analysis was not as good as it was for lipsticks and body creams, and the element concentrations were overestimated (Recoveries $>120 \%$ ). Therefore, despite a suspension preparation can be useful as a screening tool, a normalisation process is required to obtain quantitative data. One possibility would be to use a calibration curve for each target element, obtained by analysing a set of samples with a similar matrix to the unknown ones by a reference technique. Future research into the eye shadow TXRF analysis should focus on this. Our results indicated that another option to improve the results obtained for the eye shadows analysis would be to apply more sophisticated sample treatments, such as microwave digestion before the TXRF analysis. Despite this approach implying longer analysis times, the use of TXRF as a detection technique still entails lower costs than ICP-based techniques as no consumables or cooling media are necessary.

In short, the TXRF methods developed herein reveal the considerable potential for the multielement analysis of cosmetics and can be a complementary alternative to other widely used spectroscopic methods in the cosmetic analysis field. This manuscript also highlights the lack of certified reference cosmetic materials and the urgent need to develop such materials to verify the quality of the results obtained when developing analytical methods for cosmetics. 


\section{Acknowledgments}

The authors would like to acknowledge Ms. Sílvia Garro for her contribution to the experimental work. 


\section{References}

[1] S.Borowska and M.M.Brzóska, Metals in cosmetics: implications for human health, J.Appl.Toxicol. 35 (2015) 551-572.

[2] P-J.Lu, S-C.Huang, Y-P.Chen, L-C.Chiueh, D.Y-C. Shih, Analysis of titanium dioxide and zinc oxide nanoparticles in cosmetics, J.Food Drug Anal. 23 (2015) 587-594.

[3] H.Ullah, S.Noreen, Fozia, A.Rehman, A.Waseem, S.Zubair, M.Adnan, I.Ahmad, Comparative study of heavy metal content in cosmetic products of different countries marketed in Khyber Pakhtunkhwa, Pakistan, Arab. J. Chem. 10 (2017) 10-18.

[4] H.Rebiere, A.Kermaïdic, C.Ghyselinck, C.Brenier, Inorganic analysis of falsified medical products using X-ray fluorescence spectroscopy and chemometrics. Talanta 195 (2019)490-496.

[5] E.Pinto, K.Paiva, A.Carvalhido, A.Almeida, Elemental impurities in lipsticks: Results from a survey of the Portuguese and Brazilian markets. Regul. Toxicol. Pharm. 95(2018)307-313.

[6] M.A.Alnuwaiser, micro-determination and assessment of selected heavy metal ions ion foundation creams commonly used in Kingdom of Saudi Arabaia using ICP-AES. Asian J. Chem. 30(2018)27932796. [7] L.Aljerf, High-efficiency extraction of bromocresol purple dye and heavy metals as chromium from industrial effluent by adsorption onto a modified surface of zeolite: Kinetics and equilibrium study. J. Environ. Mang. 225 (2018) 120-132.

[8] M.Heep, M.Mindak, R.Cheng, Determination of total lead in lipstick: Development and validation of a microwave-assisted digestion, inductively coupled plasma-mass spectrometric method. J.Cosmet. Sci 60 (2009) 405-414.

[9] A. Salvador, A.Chisvert. Analysis of cosmetic products. Elsevier Science, 2007, 506pp, ISBN-978-0444-52260-3, DOI: https://doi.org/10.1016/B978-0-444-52260-3.X5020-7

[10] I.C.Nnormon, J.C.Igwe, C.G.Oji-mmorom, Trace metal contents of facial (make-up) cosmetics commonly used in Nigeria, Afr. J. Biotechnol. 4 (2005) 1133-1138. 
[11] M.G. Volpe, M.Nazzaro, R.Coppola, F.Rapuano, R.P. Aquino, Determination and assessments of selected heavy metals in eyeshadow cosmetics from China, Italy and USA, Microchem. J. 101 (2012) 6569.

[12] A. Salvador, M.C. Pascual-Martí, J.R. Adell, A. Requeni, J.G. March, Analytical methodologies for atomic spectrometric determination of metallic oxides in UV sunscreen creams, J. Pharmaceut. Biomed. 22 (2000) 301-306.

[13] I. Al-Saleh, S.Al-Enazi, N.Shinwari, Assessment of lead in cosmetic products, Regul. Toxicol. Pharm. 54 (2009) 105-113.

[14] E.L.Sainio, R.Jolanki, E.Hakala, L.Kanerva, Metals and arsenic in eye shadows, Contact Dermatitis 42 (2000) 5-10.

[15] O.E.Orisakwe, J.O.Otaraku, Metal concentrations in cosmetics commonly used in Nigeria, Sci. World J. 2013, Article ID 959637, 7 pages, http://dx.doi.org/10.1155/2013/959637

[16] M.A.Gondal, Z.S.Seddigi, N.M. Nasr, B.Gondal, Spectroscopic detection of health hazardous contaminants in lipstick using laser induced breakdown spectroscopy, J. Hazard. Mater. 175 (2010) 726732.

[17] A. Zakaria and Y.B. Ho, Heavy metals contamination in lipsticks and their associated health risks to lipsticks consumers. Regul. Toxicol. Pharmacol. 73 (2015) 191-195.

[18] A.A.Alqadami, M.A.Abdalla, Z.A.Alothman, K.Omer, Application of solid phase extraction on multiwalled carbon nanotubes of some heavy metal ions to analysis of skin whitening cosmetics Using ICP-AES, Int.J.Environ.Res.Public Health 10 (2013) 361-374.

[19] W.N.Chen, S.J.Jiang, Y.L.Chen, A.C.Sahayam, Slurry sampling flow injection chemical vapor generation inductively coupled plasma mass spectrometry for the determination of trace $\mathrm{Ge}, \mathrm{As}, \mathrm{Cd}, \mathrm{Sb}$, $\mathrm{Hg}$ and $\mathrm{Bi}$ in cosmetic lotions, Anal. Chim. Acta 860 (2015)8-14.

[20] T.Murphy, S.Lim, S.Huong, K.Irvine, S.Bayen, B.Kelly, K.Wilson, Application of handheld x-ray fluorescence analyzers to identify mercury in skin-whitening creams in Cambodia, J. Health Pollut, 2 (2012) 21-31. 
[21] E. DaSilva, A. Matthews David, A.Pejović-Milić, The quantification of total lead in lipsticks specimens by total reflection X-ray fluorescence spectrometry, X-Ray Spectrom. 44 (2015) 451-457.

[22] J. A.Omolaoye, A.Uzairu, C.E.Gimba, Heavy metals assessment of some eye shadows products imported into Nigeria from China. Arch. Appl. Sci. Res. 2 (2010)76-84.

[23] E. Marguí, R.Van Grieken, X-ray Fluorescence Spectrometry and Related Techniques: An Introduction. Momentum Press, New York, 2013.

[24] H.Gallardo, I.Queralt, J.Tapias, L.Candela, E.Marguí, Bromine and bromide content in soils: Analytical approach from total reflection X-ray fluorescence spectrometry. Chemosphere 156 (2016) 294301.

[25] L.Torrent, M.Iglesias, M.Hidalgo, E.Marguí, Analytical capabilities of total reflection X-ray fluorescence spectrometry for silver nanoparticles determination in soil adsorption studies. Spectrochim. Acta Part B 218 (2017) 348-355.

[26] R. Dalipi, E.Marguí, L.Borgese, L.Depero, Multi-element analysis of vegetal foodstuff by means of low power total reflection X-ray fluorescence (TXRF) spectrometry. Food Chem. 218 (2017) 348-355.

[27] E.Marguí, P.Ricketts, H.Fletcher, A.G.Karydas, A.Migliori, J.J.Leani, M.Hidalgo, I.Queralt, M.Voutchkov, Total reflection X-ray fluorescence as a fast multielemental technique for human placenta sample analysis. Spectrochim. Acta Part B 130 (2017) 53-59.

[28] C.García-Florentino, M. Maguregui, E. Marguí, L. Torrent, I. Queralt, J.M. Madariaga, Development of total teflection X-ray fluorescence spectrometry quantitative methodologies for elemental characterization of building materials and their degradation products. Spectrochim. Acta Part B $143(2018) 18-25$.

[29] E.Marguí, I.Queralt, M.Hialgo, Determination of platinum group metal catalyst residues in active pharmaceutical ingredients by means of total reflection X-ray spectrometry, Spectrochim. Acta Part B 86 (2013) 50-54.

[30] Certificate RM 619 (Body cream), Netherlands Food and Consumer Product Safety Authority. Ministry of Economic Affairs, http://145.12.37.100/txmpub/files/?p_file id=2209017 (Accessed February 2019) 
[31] I. De La Calle, N.Cabaleiro, V. Romero, I. Lavilla, C. Bendicho, Sample pretreatment strategies for total reflection X-ray fluorescence analysis: A tutorial review, Spectrochim. Acta Part B 90 (2013) 23-54.

[32] E.Marguí, M.Sagué, I.Queralt, M.Hialgo, Liquid phase mictroextraction strategies combined with total reflection X-ray spectrometry for the determination of low amounts of inorganic antimony species in waters. Anal. Chim. Acta 786 (2013) 8-15.

[33] E.Marguí, I.Queralt, M.Hidalgo. Determination of cadmium at ultratrace levels in environmental water samples by means of total reflection X-ray spectrometry after dispersive liquid-liquid microextraction. J. Anal. At. Spectrom. 28 (2013) 266-273.

[34] E. Marguí, J. Jablan, M.Gerić, S. Inić, A-M. Domijan, R.Janušić, B. Šarčević, I.Queralt, V.GarajVrhovac, Critical evaluation of the use of total reflection X-ray fluorescence spectrometry for the analysis of whole blood samples: application to patients with thyroid gland diseases, Anla. Bioanal. Chem, In press (https://doi.org/10.1007/s00216-019-01618-3).

[35] E. Marguí, G. H. Floor, M. Hidalgo, P. Kregsamer, G. Román-Ross, C. Streli, I. Queralt, Analytical possibilities of total reflection X-ray spectrometry (TXRF) for trace selenium determination in soils, Anal. Chem. 82 (2010) 7744-7751.

[36] E.Marguí, J.C.Tapias, A.Casas, M.Hidalgo, I.Queralt, Analysis of inlet and outlet industrial wastewater effluents by means of benchtop total reflection X-ray fluorescence spectrometry, Chemosphere 80 (2010) 263-270.

[37] R.Dalipi, E.Marguí, L.Borgese, F.Bilo, L.E.Depero, Analytical performance of benchtop total reflection X-ray fluorescence instrumentation for multielemental analysis of wine samples, Spectrochim. Acta Part B 120 (2016) 37-43.

[38] Kosmetische Mittel: BrR empfiehlt Schwermetallgehalte über Reinheitsandforderungen der Ausgangsstoffe zu regeln.

https://mobil.bfr.bund.de/cm/343/kosmetische mittel bfr_empfiehlt schwermetallgehalte ueber.pdf (Accessed February 2019).

[39] Cosumer Product Safety: Guidance on heavy metal impurities in cosmetics. 
https://www.canada.ca/en/health-canada/services/consumer-product-safety/reports-publications/industryprofessionals/guidance-heavy-metal-impurities-cosmetics.html.(Accessed February 2019).

[40] DE UTR200 - Powerful Ultrasonic Cup Horn, https://www.hielscher.com/utr2_p.htm (Accessed February 2019).

[41] P.Howe, P.Watts, Tin and Inorganic tin compounds, World Health Organization (WHO), 2005.

[42] E.Marguí, P.Kregsamer, M.Hidalgo, J.Tapias, I.Queralt, C.Streli, Analytical approaches for $\mathrm{Hg}$ determination in wastewater samples by means of total reflection X-ray fluorescence spectrometry, Talanta 82 (2010) 821-827.

[43] E. Marguí, I. Queralt, M. Guerra, N. Kallithrakas-Kontos, Mercury determination at trace levels using membrane preconcentration and benchtop total reflection X-ray fluorescence analysis, Spectrochim. Acta Part B 149 (2018)84-90. 
Table 1. Summary of the analytical details of representative publications about the analyses of lipsticks, eye shadows and body creams.

\begin{tabular}{|c|c|c|c|c|c|c|c|c|}
\hline & Technique & Cosmetic type & Analyte & $\begin{array}{c}\text { Sample } \\
\text { amount(g) }\end{array}$ & Sample treatment & $\begin{array}{c}\mathrm{LOD} \\
\left(\mathrm{mg} \mathrm{Kg}^{-1}\right)\end{array}$ & $\begin{array}{c}\text { Concentration } \\
\text { range } \\
\left(\mathrm{mg} \mathrm{Kg}^{-1}\right)\end{array}$ & Ref. \\
\hline \multirow{6}{*}{ AAS } & \multirow{3}{*}{ FAAS } & Lipstick & $\mathrm{Pb}, \mathrm{Cd}, \mathrm{Cr}, \mathrm{Zn}, \mathrm{Ni}, \mathrm{Fe}$ & 1 & Digestion $\left(\mathrm{HNO}_{3}+\mathrm{HClO}_{4}\right)$ & n.r. & $<1-250$ & {$[10]$} \\
\hline & & Eye shadow & $\mathrm{Pb}$ & 1 & Digestion $\left(\mathrm{HNO}_{3}+\mathrm{HF}\right)$ & n.r. & $0.25-81.5$ & [11] \\
\hline & & Sunscreen & $\mathrm{Zn}, \mathrm{Fe}$ & $0.04-0.2$ & Emulsification (IBMK+Nemol K-39) & $\overline{0.03}$ & $0.1-4$ & [12] \\
\hline & \multirow{3}{*}{ ETAAS } & Lipstick & $\mathrm{Pb}$ & 0.2 & Digestion $\left(\mathrm{HNO}_{3}+\mathrm{H}_{2} \mathrm{O}_{2}\right)$ & $<1$ & $0.2-3760$ & [13] \\
\hline & & Eye shadow & $\mathrm{Pb}, \mathrm{Cr}, \mathrm{As}, \mathrm{Co}, \mathrm{Ni}$ & 0.15 & Digestion $\left(\mathrm{HNO}_{3}+\mathrm{HCl}\right)$ & 0.3 & Up to 272 & [14] \\
\hline & & Cream & $\mathrm{Pb}, \mathrm{Ni}$ & 0.2 & Digestion $\left(\mathrm{H}_{2} \mathrm{SO}_{4}\right)$ & 0.2 & $1.1-45.9$ & [15] \\
\hline \multirow{7}{*}{ ICP } & \multirow{4}{*}{ ICP-OES } & Lipstick & $\mathrm{Pb}, \mathrm{Cr}, \mathrm{Cd}, \mathrm{Zn}$ & 1 & Digestion $\left(\mathrm{HNO}_{3}+\mathrm{H}_{2} \mathrm{O}_{2}\right)$ & n.r. & $10-20$ & {$[16]$} \\
\hline & & & $\mathrm{Pb}, \mathrm{Cd}, \mathrm{Cr}$ & 0.4 & MW Digestion $\left(\mathrm{HNO}_{3}\right)$ & $0.06-0.63$ & $0.06-15.44$ & {$[17]$} \\
\hline & & Cream & $\mathrm{As}, \mathrm{Bi}, \mathrm{Cd}, \mathrm{Pb}, \mathrm{Hg}, \mathrm{Ti}$ & $0.1-0.25$ & MW Digestion $\left(\mathrm{HNO}_{3}+\mathrm{H}_{2} \mathrm{O}_{2}+\mathrm{HF}\right)$ & n.r. & $0.3-4.8$ & [18] \\
\hline & & Sunscreen & $\mathrm{Ti}$ & 0.15 & MW Digestion $\left(\mathrm{HNO}_{3}+\mathrm{HCl}\right)$ & 0.035 & $5000-12500$ & [12] \\
\hline & \multirow{3}{*}{ ICP-MS } & Lipstick & $\mathrm{Pb}$ & 0.3 & MW Digestion $\left(\mathrm{HNO}_{3}+\mathrm{HF}\right)$ & 0.04 & 1.07 (average) & {$[8]$} \\
\hline & & Eye shadow & $\mathrm{Cd}, \mathrm{Co}, \mathrm{Cr}, \mathrm{Ni}$ & 1 & MW Digestion $\left(\mathrm{HNO}_{3}+\mathrm{HF}\right)$ & n.r. & $0.6-4124$ & [11] \\
\hline & & Cream & $\mathrm{Ge}, \mathrm{As}, \mathrm{Cd}, \mathrm{Sb}, \mathrm{Hg}, \mathrm{Bi}$ & 0.2 & $\begin{array}{l}\text { Slurry (Thiourea+Cysteine+Triton } \\
\mathrm{X} 100+\mathrm{HCl} \text { ) }\end{array}$ & $<0.3$ & $0.3-5.5$ & [19] \\
\hline LIBS & & Lipstick & $\mathrm{Pb}, \mathrm{Cr}, \mathrm{Cd}, \mathrm{Zn}$ & n.r. & Frozen and cut into small pieces $(\varnothing: 1 \mathrm{~cm})$ & $1.7-2.6$ & $10-20$ & {$[16]$} \\
\hline \multirow{5}{*}{ XRF } & EDXRF & Eye shadow & $\begin{array}{l}\mathrm{K}, \mathrm{S}, \mathrm{Cl}, \mathrm{Al}, \mathrm{Si}, \mathrm{Ca}, \mathrm{Ti}, \mathrm{Mn}, \\
\mathrm{Fe}, \mathrm{Pb}, \mathrm{Zn}, \mathrm{Rb}, \mathrm{Bi}\end{array}$ & 0.3 & Pellet (diluted with boric acid 1:1) & n.r. & $209-257706$ & [7] \\
\hline & $\begin{array}{l}\text { Handheld- } \\
\text { EDXRF }\end{array}$ & Skin cream & $\mathrm{Hg}$ & $3 .-5^{\mathrm{a}}$ & Deposition on a Mylar film & 20 & $20-23000$ & {$[20]$} \\
\hline & \multirow{3}{*}{ TXRF } & & & & & & & \\
\hline & & Lipstick & $\mathrm{Pb}$ & $0.1-0.4$ & Liquefication / solidification steps & 0.04 & $0.4-5.6$ & {$[21]$} \\
\hline & & $\begin{array}{l}\text { Lipstick, eye } \\
\text { shadows, body } \\
\text { creams }\end{array}$ & $\begin{array}{l}\text { K, Ca, Ti, Mn, Fe, } \mathrm{Cu}, \mathrm{Zn}, \\
\mathrm{Rb}, \mathrm{Sr}, \mathrm{Ba}, \mathrm{Sn}, \mathrm{Pb}, \mathrm{Cd}, \mathrm{Cr} \\
\mathrm{Ni}, \mathrm{Sb}, \mathrm{Se}\end{array}$ & $0.01-0.02$ & Solid suspension & $\begin{array}{l}\text { Lipsticks: } 2 /(100)^{\mathrm{b}} \\
\text { Eye shadows: } 5 /(150)^{\mathrm{b}} \\
\text { Creams: } 1-8\end{array}$ & $>10000$ & $\begin{array}{l}\text { This } \\
\text { work }\end{array}$ \\
\hline
\end{tabular}

n.r.: not reported, ${ }^{\mathrm{a}} \mathrm{mL},{ }^{\mathrm{b}}$ in brackets, LODs for light elements $(\mathrm{K}, \mathrm{Ca})$ 
Table 2. Summary of the procedure followed to prepare the cosmetic sample suspensions for the TXRF analysis

\begin{tabular}{|c|c|c|c|c|c|c|}
\hline Cosmetic & Amount & Solvent $^{\mathrm{a}}$ & IS $^{b}$ & Homogenisation & Deposition & Drying mode \\
\hline type & (mg) & & & & volume $(\mu \mathrm{L})$ & on the reflector \\
\hline Lipsticks & 10 & Chloroform & Mo (organic matrix) & 5 min sonication & 5 & Room T \\
\hline Eye shadows & 20 & Triton $1 \%$ & Pd (aqueous matrix) & 5 min sonication & 5 & IR lamp \\
\hline Creams & 20 & Triton $1 \%$ & $\operatorname{Pd}$ (aqueous matrix) & 5 min sonication & 5 & IR lamp \\
\hline
\end{tabular}

${ }^{\mathrm{a}}$ In all cases: volume is $1 \mathrm{~mL},{ }^{\mathrm{b}} \mathrm{In}$ all cases: $50 \mu \mathrm{L}$ of $200 \mathrm{mg} \cdot \mathrm{L}^{-1} \mathrm{IS}$ solution 
Table 3. Results obtained by determining trace elements in the reference material RM 619 Body cream (Netherlands Food Consumer Product Safety Authority) by the developed TXRF method. Results are expressed as the mean concentration values of three replicates with the associated standard deviation.

TXRF method

Element Reference value ( $\left.\mathrm{mg} \mathrm{kg}^{-1}\right)$

$\begin{array}{llll}\text { TXRF value (mg kg-1) } & \text { Recovery (\%) } & \text { RSD (\%) } & \text { LOD }\left(\mathrm{mg} \mathrm{kg}^{-1}\right)\end{array}$

\begin{tabular}{|c|c|c|c|c|c|}
\hline $\mathrm{Pb}$ & $11.8 \pm 0.81$ & $16 \pm 2$ & 130 & 12 & 2.6 \\
\hline $\mathrm{Cd}$ & $46.7 \pm 3.5$ & $52 \pm 1$ & 111 & 2 & 1.6 \\
\hline $\mathrm{Cr}$ & $47.8 \pm 0.81$ & $57 \pm 7$ & 119 & 12 & 8.3 \\
\hline $\mathrm{Ni}$ & $49.2 \pm 4.4$ & $50 \pm 4$ & 102 & 8 & 3.1 \\
\hline $\mathrm{Hg}$ & $2.33 \pm 0.81$ & n.d. & - & - & - \\
\hline $\mathrm{Sb}$ & $31.1 \pm 5.0$ & $29 \pm 3$ & 93 & 10 & 2.8 \\
\hline $\mathrm{Se}$ & $44.0 \pm 3.6$ & $39 \pm 2$ & 89 & 5 & 1.9 \\
\hline
\end{tabular}




\section{FIGURE CAPTIONS}

Fig. 1. TXRF spectra obtained in the analysis of the different coloured lipstick samples.

Fig. 2. Effect of measurement time on the repeatability of $\mathrm{Rb}$ (trace element, $\sim 60 \mathrm{mg} / \mathrm{kg}$ ) and $\mathrm{Ti}$ (minor element, $\sim 6000 \mathrm{mg} / \mathrm{kg}$ ) results for the analysis of lipsticks by the suspension + TXRF analysis. Experimental tests were performed using the red lipstick sample.

Fig. 3. TXRF and ICP results obtained by the analysis of different coloured lipstick sampless: (A) Purple, (B) Red, (C) Brown, (D) Orange. Results are expressed as the mean concentration values of three replicates.

Fig. 4. 2D mappings for $\mathrm{Cl}, \mathrm{K}, \mathrm{Ca}$ and $\mathrm{Ti}$ in a $5 \mu \mathrm{L}$ pink eye shadow sample spot in a quartz glass reflector, prepared by suspending $20 \mathrm{mg}$ of sample in $1 \mathrm{~mL}$ Triton \% without sonication before deposition (A) and with 5-minute conventional sonication (B). The colour scale is expressed in $\mu \mathrm{g} \mathrm{cm}^{-2}$ (measurement details: $10 \mathrm{kV}, 1 \mathrm{~mA}, 200 \mathrm{~s}, 0.3 \mathrm{~mm}, 15 \mathrm{x} 15 \mathrm{map}$ ).

Fig. 5. TXRF and ICP results obtained by analysing the different coloured eye shadow samples: (A) Pink, (B) Orange, (C) Brown, (D) Blue. Results are expressed as the mean concentration values of three replicates.

Fig. 6. TXRF spectrum obtained by analysing the body cream reference material CHEK RM 619 (A) and a commercial facial cream (B). In both cases, Pd was used as the internal standard for quantification purposes. 
Fig. 1.

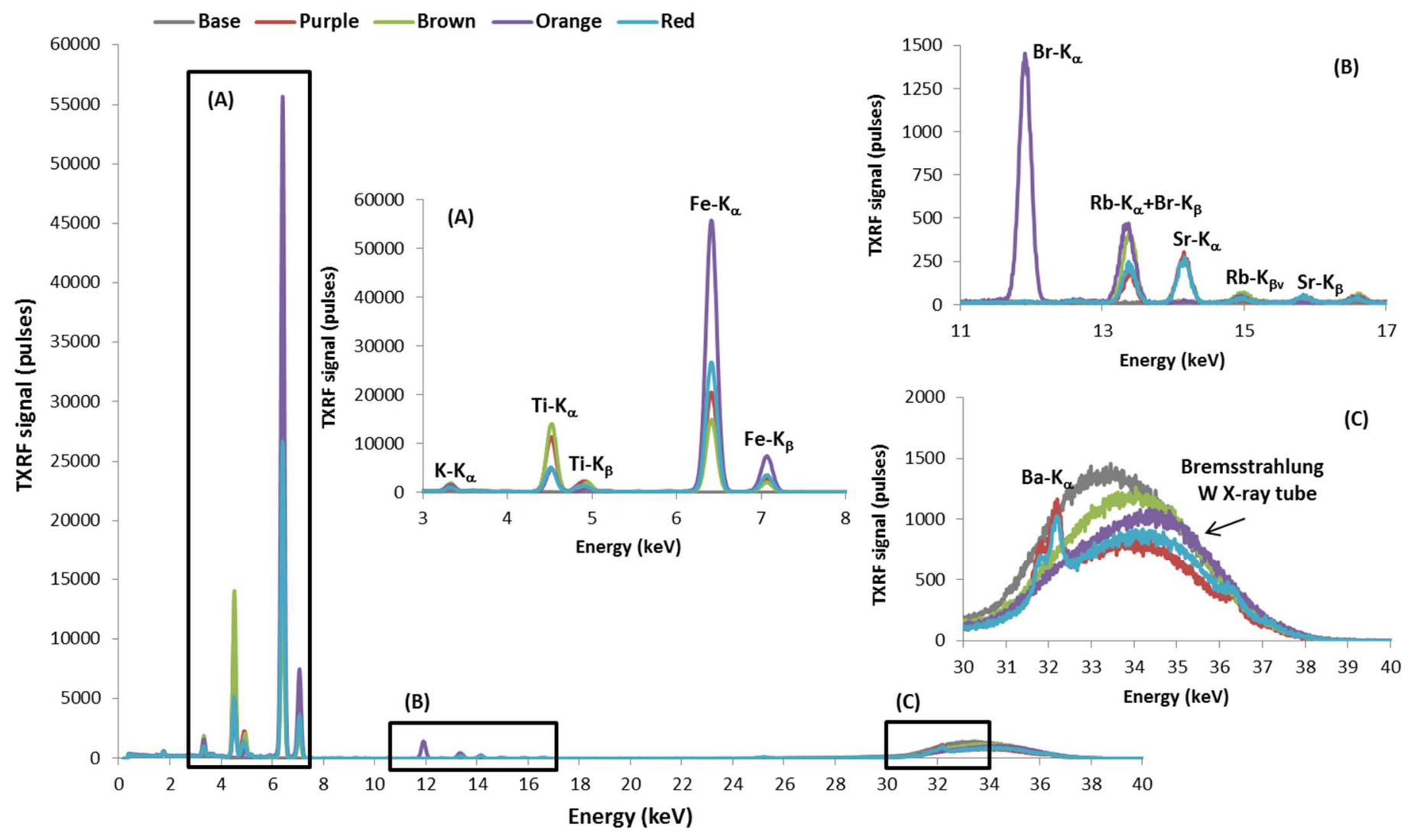


Fig. 2.

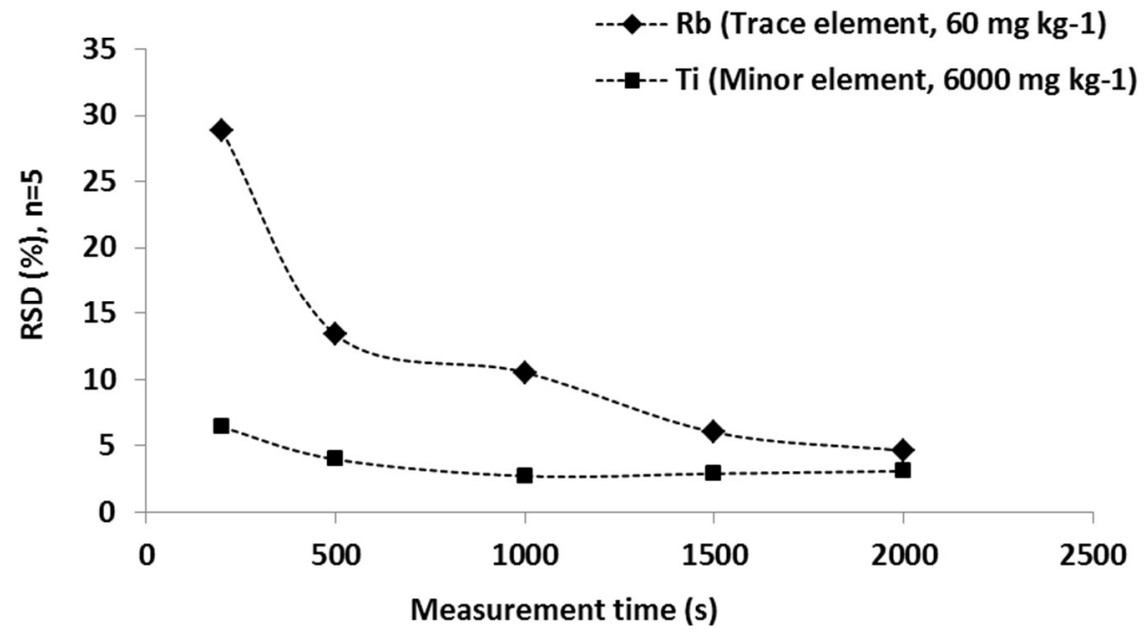


Fig. 3.
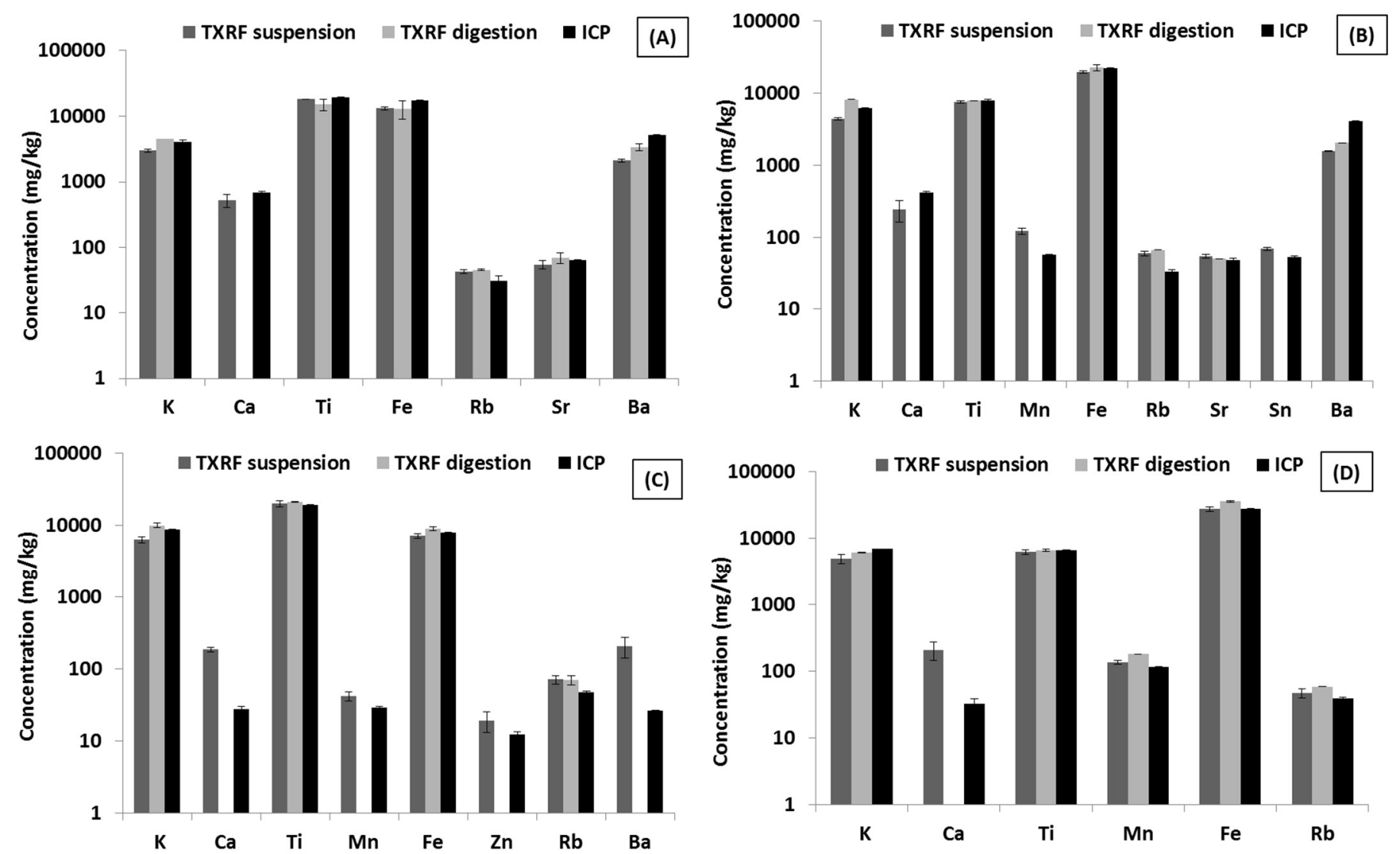
Fig. 4.

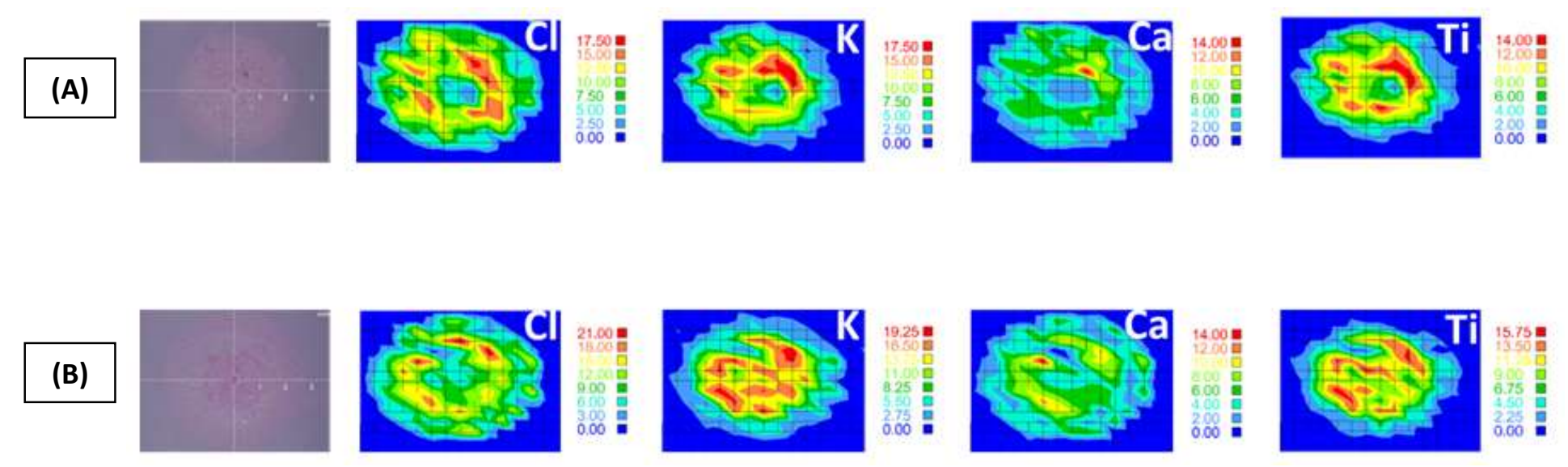


Fig. 5.
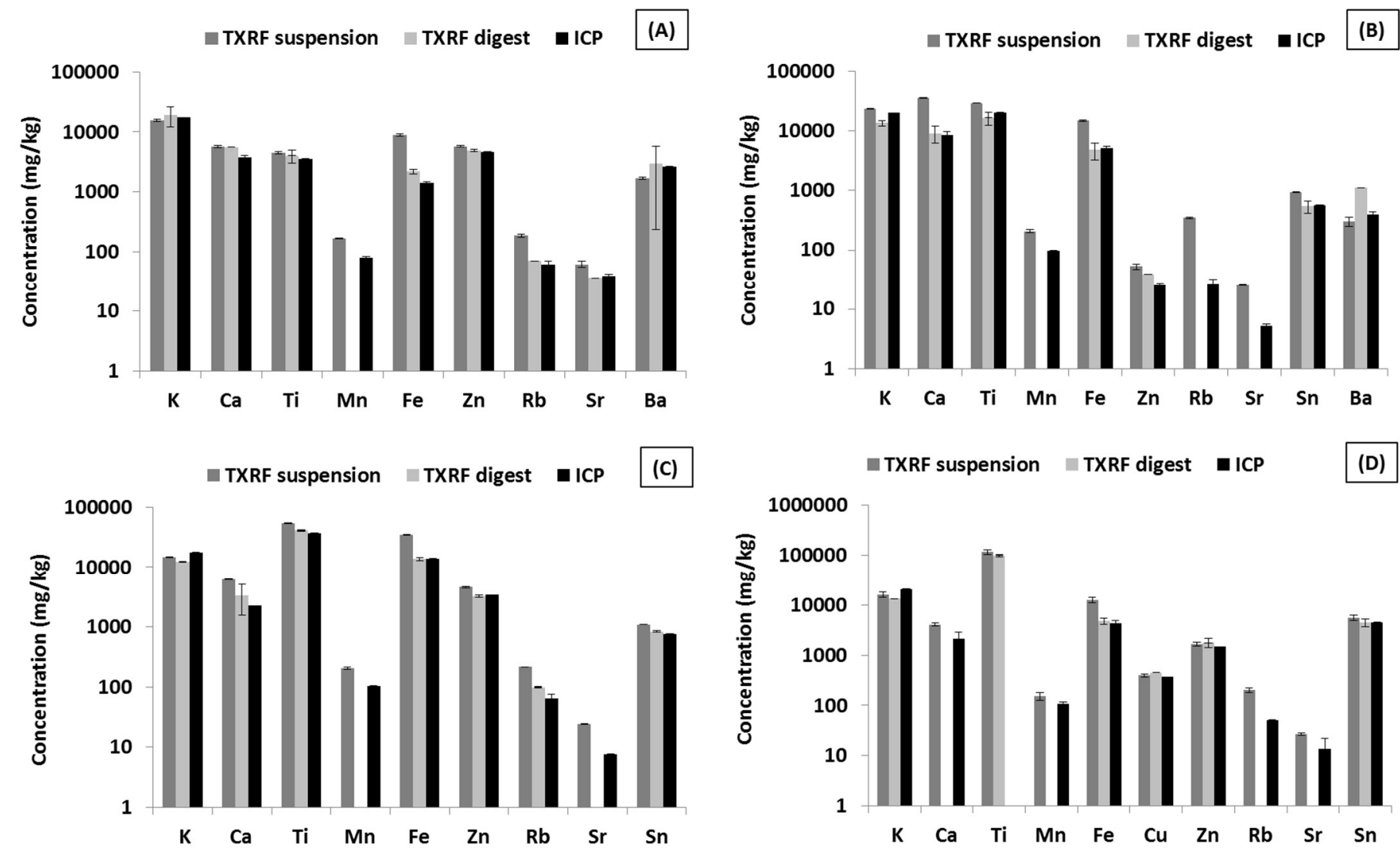
Fig. 6.
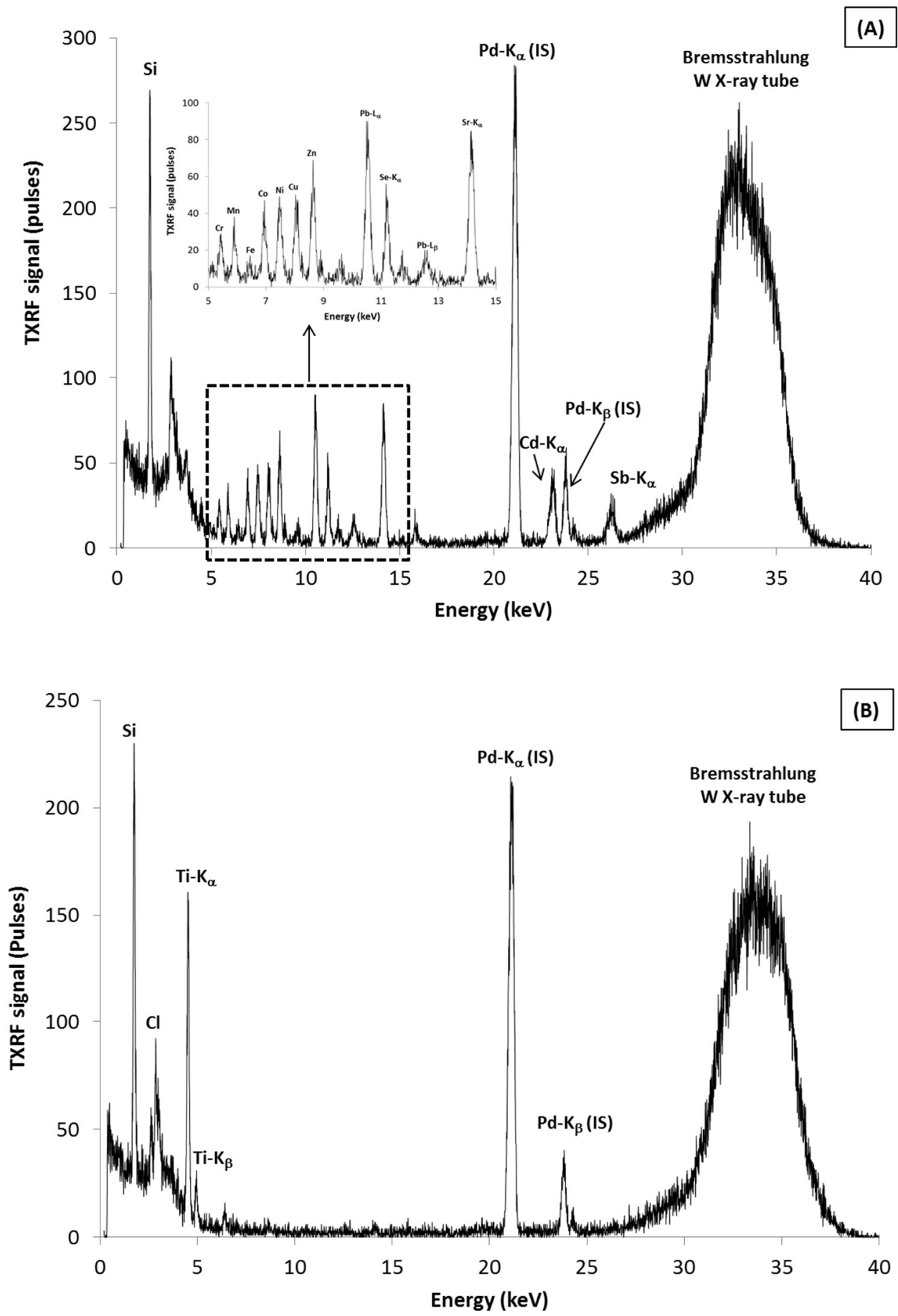